\title{
Upgrades of the ATLAS Muon Spectrometer with New Small-Diameter Drift Tube Chambers
}

\author{
O. Kortner ${ }^{\mathrm{a}}$, H. Kroha ${ }^{\mathrm{a}, *}$, S. Nowak ${ }^{\mathrm{a}}$, S. Podkladkin ${ }^{\mathrm{a}}$, P. Rieck ${ }^{\mathrm{a}}$, K. Schmidt-Sommerfeld ${ }^{\mathrm{a}}$, E. Takasugia ${ }^{\mathrm{a}}$, V. Walbrecht ${ }^{\mathrm{a}}$, \\ R. Fakhrutdinov ${ }^{\text {b }}$ A. Kozhin ${ }^{\text {b }}$ \\ ${ }^{a}$ Max-Planck-Institute for Physics, Munich, Germany \\ ${ }^{b}$ Institute for High Energy Physics, Protvino, Russia
}

\begin{abstract}
Small diameter muon drift-tube (sMDT) chambers with $15 \mathrm{~mm}$ tube diameter provide excellent spatial resolution like the MDT chambers with $30 \mathrm{~mm}$ tube diameter used in the ATLAS muon spectrometer so far, but can be operated at ten times higher background rates and allow for the instrumentation of regions where MDT chambers do not fit in. In April 2014 two BME and in January 2016 another 12 BMG sMDT chambers have been installed in the middle layer of the barrel muon detector and are operational since then in order to increase the acceptance for precision muon momentum measurement using three chamber layers. An unprecedently high sense wire positioning accuracy of $5 \mu \mathrm{m}$ (rms) has been achieved. In the next long LHC shutdown 2019-2020, 16 BIS 78 sMDT chambers will be installed in the barrel inner layer in the transition region to inner endcap layer to make room for the installation of new RPC muon trigger chambers in order to reduce the accidential trigger rate in this region as required for operation at HL-LHC. This is a pilot project for the complete replacement of the MDT chambers in the small sectors of the barrel inner layer by integrated sMDT-RPC detectors (BIS 1-6) in the ATLAS upgrade for HL-LHC.
\end{abstract}

Keywords: Muon precision tracking, ATLAS detector, drift-tube detectors, MDT chambers, sMDT chambers PACS: 29.40.Cs, 29.40.Gx

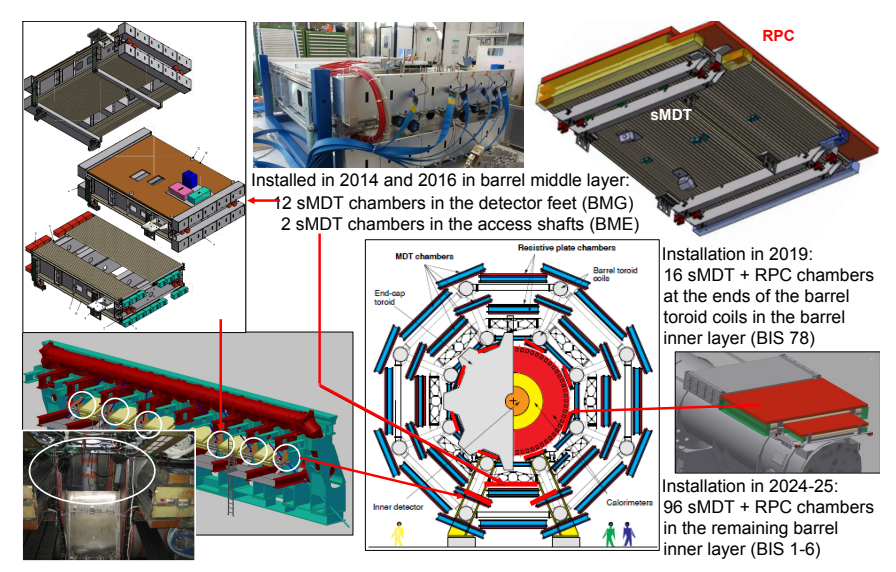

Figure 1: Overview of the sMDT chambers in the ATLAS muon spectrometer.

\section{The sMDT Chambers in ATLAS}

An overview of the small-diameter drift tube (sMDT) chambers contructed for the ATLAS muon spectrometer is given in Figure 1. The first sMDT chambers [1] have been installed in April 2014 in the barrel middle layer in the access shafts to the calorimeters at the bottom of the detector (BME chambers), followed in January 2016 by the installation of 12 BMG sMDT

\footnotetext{
${ }^{*}$ Corresponding author

Email address: kroha@mppmu.mpg.de (H. Kroha)
}

chambers in gaps inside the detector feet in the bottom sectors of the barrel middle layer. Both the BME and the BMG chambers improve the muon momentum resolution by a factor of two by allowing for three-point track curvature measurement within their geometrical acceptance. Currently 16 BIS 78 sMDT chambers are under construction for installation at the ends of the toroid coils in small sectors of the barrel inner layer in the long LHC shutdown 2019-2020. The chambers will replace the present BIS 7 and BIS 8 MDT chambers to make space for new thin-gap RPC trigger chambers which will significantly reduce the accidental muon trigger rate in the transition region $1.0<|\eta|<1.3$ between barrel and endcap at HL-LHC. In the ATLAS high-luminosity upgrade 2025-2026, the remaining MDT chambers in the small sectors of the barrel innner layer (BIS 1-6) will be replaced by integrated sMDTRPC chambers of the same type as the new BIS 78 chambers in order to improve the muon trigger coverage and efficiency in the barrel at the High-Luminosity LHC (HL-LHC) [2].

\section{2. sMDT Chamber Design and Construction}

Small-diameter muon drift tube (sMDT) chambers with a tube diameter of $15 \mathrm{~mm}$, i.e. half the diameter of the MDT tubes, have been developed to cope with the higher background irradiation rates at High-Luminosity LHC (HL-LHC) and future hadron colliders and to fit into the small available spaces available for the upgrades of the ATLAS muon spectrometer. 


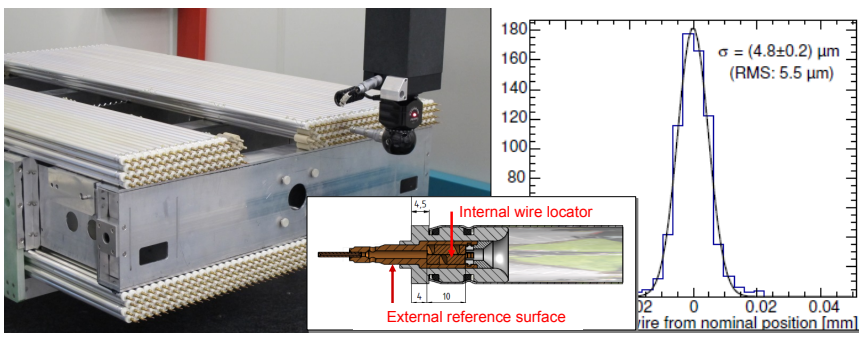

Figure 2: Left: Measurement of the sense wire positions of a BMG sMDT chamber with a coordinate measuring machine. The endplug design allowing for precise wire positioning and mechnical measurement is also shown. Right: Residual distribution of the measured wire positions in a BMG chamber with respect to the nominal wire grid.

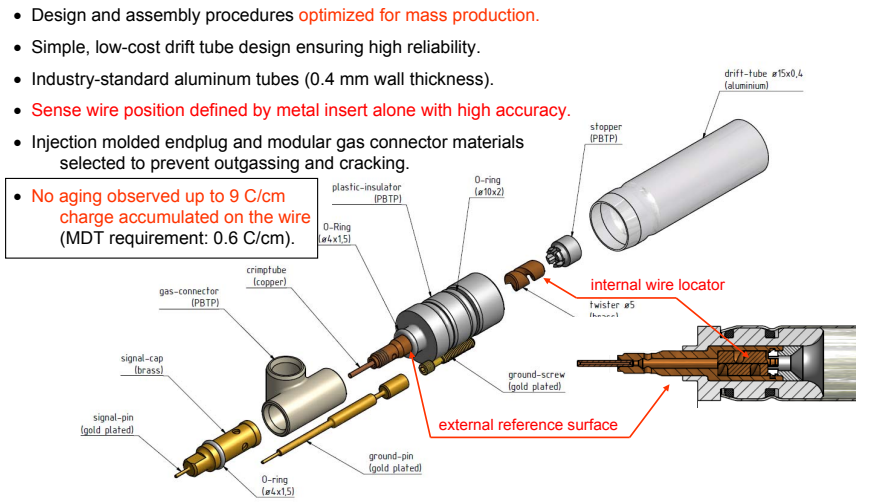

Figure 3: Exploded view of an SMDT drift tube. The brass inserts of the injection molded pastic endplugs hold the sense wires and define their position in the chamber with few micron accuracy. The gas, signal and ground connections are also shown.

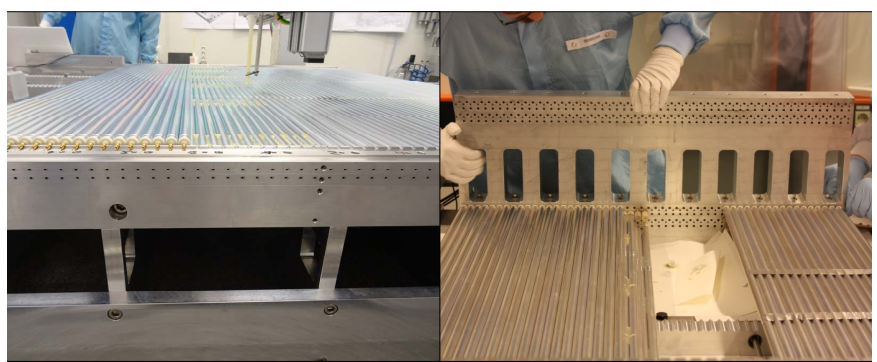

Figure 4: sMDT assembly jigs (left: BIS 78, right: BMG) with precise hole matrix for postioning of the endplug reference surfaces and, therefore, of the sense wires. A multilayer of drift tubes can be completed within one working day. The multilayer distance is alos precisely determined by the jig.

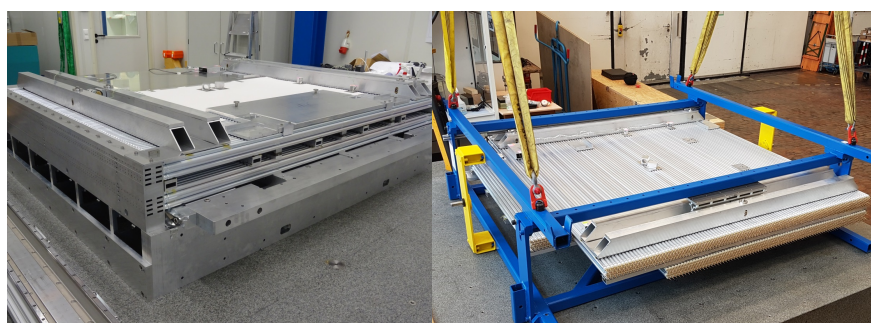

Figure 5: BIS 78 sMDT chamber (left: completed on the assembly table, right: in the transport frame).

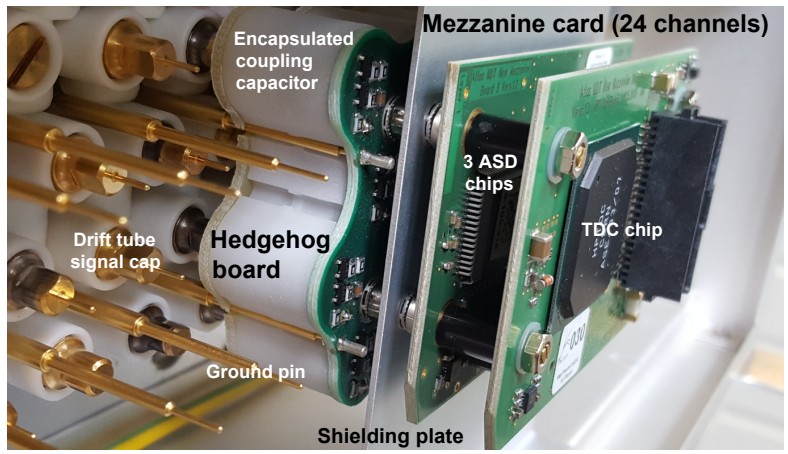

Figure 6: Stacked readout boards of sMDT chambers carrying three octal Amplifier-Shaper-Dicriminator chips and one TDC chip. The coupling capacitors to each drift tube are encapsulated in plastic tubes for protection against discharges. The so called hedgehog part with the receptacles for $6 \times 4$ signal and $6 \times 3$ ground pins inserted in the triangular gaps between adjacent tube walls contains discharge protection circuits for all ASD channels.

They have an order of magnitude higher rate capability than the MDT chambers [1].

At the same time, the drift tube design and the chamber assembly methods have been optimised for mass production leading to significant savings in cost, construction time and manpower compared to the ATLAS MDT chambers while providing even higher sense wire positioning accuracy. A record sense wire positioning accuracy of better than $5 \mu \mathrm{m}$ (rms) has been achieved for the BMG chambers with about 350 drift tubes of $1.12 \mathrm{~m}$ length [1]. The wire positioning accuracy of the large BIS 78 chambers with up to 744 drift tubes of $1.66 \mathrm{~m}$ length is better than $10 \mu \mathrm{m}$ (rms). The required positioning accuracy is $20 \mu \mathrm{m}$. The wire positions at the tube ends are measured with few micron precision with a coordinate measuring machine (CMM) for the BMG chambers (see Figure 2) and with a feeler gauge on a flat granite table for the BIS 78 chambers. The chambers carry several optical monitoring systems for deformation measurements and global alignment which are mounted with high accuracy with respect to the wires during chamber assembly and are measured withy $10 \mu \mathrm{m}$ precision with the CMM or with feeler gauges, respectively.

The complex chamber shapes required for the ATLAS upgrades can only be realised with the specific sMDT drift tube design and assembly procedure (see Figures 3 and 4). Standard aluminium tubes with $0.4 \mathrm{~mm}$ wall thickness are used. The sMDT chambers are operated with the same $\mathrm{Ar}: \mathrm{CO}_{2}$ (93:7) gas mixture at 3 bar and the same gas gain of 20000 as the MDT chambers. Assembled BIS 78 sMDT chambers are shown in Figure 5. Dedicated stacked readout electronics boards have been developed for the sMDT chambers which carry the same ASD and TDC chips as the MDT chambers (see Figure 6).

\section{References}

[1] H. Kroha, et al., Construction and Test of New Precision Drift Tube Chambers for the ATLAS Muon Spectrometer, Nucl. Instr. and Meth. A 845 (2017) 244

[2] The ATLAS collaboration, Technical Design Report for the Phase-II Upgrade of the ATLAS Muon Spectrometer, CERN-LHCC-2017-017, December 2017. 